\title{
Stepwise reductions in US density and intensity: Maintenance and extinction of conditioned suppression
}

\author{
D. CHRIS ANDERSON and CHARLES R. CROWELL \\ University of Notre Dame, Notre Dame, Indiana
}

and

\author{
RAPHAEL RAMIREZ \\ State University of New York at Stony Brook, Stony Brook, New York
}

\begin{abstract}
Information was sought on how the strength of fear, acquired and extinguished in a classical conditioning paradigm, might be affected by certain of the circumstances that normally are associated with the course of avoidance learning. Following preconditioning exposures to the conditioned stimulus, one group of rats (Group PRF) was given continuous conditioned stimulusunconditioned stimulus (CS-US) acquisition trials followed by stepwise reductions in US probability (given the CS) over three phases to about $11 \%$ for the final phase. Another group, Group US(lo), was given continuous CS-US pairings throughout, but, following acquisition, received stepwise reductions in US intensity (to permit evaluation of a progressively changing feature of the US over a wide range without having to employ excessive shock) over the same phases. A third group, Group US(hi), received unchanging (from acquisition) CS-US pairings over these phases, and an explicitly unpaired control group (Group RU) was included. Although outcomes differed somewhat depending upon whether suppression ratios or absolute measures of responding were considered, the major findings were that suppression to the CS was complete by the end of acquisition and persisted thereafter throughout the three phases for all but control subjects. In contrast to Group US(lo), which showed relatively little resistance to extinction of suppression to subsequent CS-only exposures, Groups US(hi) and PRF displayed marked resistance over two separate, extended sets of extinction sessions. Suppression to context cues was pronounced only for Groups US(hi) and RU, and varied as a function of US parameters and not whether the shocks were or were not signaled. Theoretical reconciliation of these findings was most difficult for Group PRF.
\end{abstract}

The concept of acquired fear, which is generally presumed to result from classical conditioning procedures (Brown, 1961; Kimble, 1961), has been widely invoked in accounts of signaled avoidance learning (Mowrer, 1947, 1960; Rescorla \& Solomon, 1967). To many, the paradigmatic similarities of fear-conditioning and avoidance-learning procedures have been a sufficient basis for theoretical speculation involving common explanatory mechanisms. Thus, it has been postulated that fear becomes conditioned to the signaling stimulus early in avoidance training as a result of pairings with shock, and then serves motivationally to energize and reinforce avoidance responding.

Clearly, such theorizing depends critically upon implicit assumptions about the course of fear acquisition and extinction during avoidance learning, not to mention suppositions regarding the possible interaction of fear and

\footnotetext{
Thanks are due Christopher Cunningham and Judson S. Brown for their helpful suggestions regarding the drafting and theoretical aspects of this manuscript. Send reprint requests to D. Chris Anderson, Department of Psychology, 112 Haggar Hall, University of Notre Dame, Notre Dame, IN 46556.
}

avoidance behavior. However, it is not commonly recognized that two-factor accounts of avoidance learning really rest upon two separate kinds of assumptions about fear acquisition and extinction. On the one hand, two-factor theory implies that fear would develop and be maintained in a classical conditioning paradigm in which parameters were fashioned after features of the conditioned stimulus (CS) and unconditioned stimulus (US) found in the avoidance procedure, that is, a paradigm in which parameters were such that both CS and US durations were variable and were likely to decrease progressively over trials, and in which the US did not necessarily occur on every trial. On the other hand, two-factor theory clearly presumes that fear does in fact develop, and is maintained, in the avoidance paradigm per se. Although closely related, these two implicit assumptions regarding fear acquisition and extinction are conceptually distinct. A demonstration of fear acquisition and maintenance in a conditioning paradigm, even with parameters made to resemble characteristics of the avoidance procedure, does not necessarily ensure that fear is present and operative during avoidance learning. This is primarily true because other critical 
differences between conditioning and avoidance paradigms, such as the avoidance response itself, may alter the acquisition, maintenance, or role of conditioned fear in the avoidance paradigm.

The weight of experimental investigation, to date, has been directed toward analysis of the presumed role of conditioned fear within the avoidance paradigm itself. For the most part, the relation between fear and avoidance responding therein has been found to be complicated, at best (e.g., Kamin, Brimer, \& Black, 1963; Linden, 1969; Starr \& Mineka, 1977). For example, Kamin et al. (1963) found little difference among the response latencies of groups of rats that were required to make 3,9 , or 27 consecutive avoidance trials, but nonetheless found a large difference in the magnitude of fear exhibited to the avoidance signal (CS) in an independent responsesuppression test. The group trained to 27 avoidances showed significantly less suppression, and presumably less fear, than did the other two groups (which were equally suppressed). Linden (1969) and Mineka and her colleagues (Mineka, 1985; Mineka, Cook, \& Miller, 1984; Starr \& Mineka, 1977) have shown further that the apparent reduction in fear with extended avoidance training is probably due to inhibitory properties that accrue to the avoidance response itself.

The purpose of the present study was to investigate the acquisition and maintenance of fear in a conditioning paradigm in which the parameters resemble some of the features of the CS and US that are found in avoidance training. The purpose here was not to assess the role of fear in avoidance learning, but rather to examine an unheralded assumption implicit in a historically important account of avoidance phenomena. Specifically, the consequences for fear acquisition and extinction of two different, avoidance-like conditioning regimens were examined: a gradual change from continuous (CS-US pairing on every trial) to partial reinforcement (US omitted on some trials) with a fixed US intensity, and a gradual change from high to low US intensity with a fixed schedule of reinforcement. US intensity was varied, instead of US duration, in an effort to evaluate a progressively changing feature of the US over a wide range without having to utilize excessive amounts of shock. The progressive partial reinforcement procedures examined in this study differed from the fixed schedules more commonly employed and, at least in this respect, were more comparable to procedures used by Starr and Mineka (1977) in their study of avoidance learning.

\section{METHOD}

\section{Subjects}

Twenty-four naive male albino Sprague-Dawley rats, 90-110 days of age, were purchased from Charles River Laboratories, Inc. Each was caged singly in an environment with unchanging light, temperature, and ambient background conditions. Following acclimation, body weights were reduced through water deprivation to $80 \%$ of ad lib values determined immediately before water removal. They were maintained thereafter at these weights $( \pm 2 \%)$ on a $23-\mathrm{h}, 55-\mathrm{m}$ ( \pm 2-3 min) water-deprivation schedule. Lab chow was available ad lib.

\section{Apparatus}

Three identical operant chambers (Gerbrands, Model 4C), housed in separate sound- and light-controlled, ventilated environments, were used. A 7.5-W bulb was mounted on the inner wall of each isolation chamber, $25 \mathrm{~cm}$ above the floors of the operant boxes. Each chamber was constructed with two metal sides, a grid floor, and two sides and a hinged top of Plexiglas. Protruding through one metal side was a standard rat bar and, nearby, a stainless steel cup through which single drops of water could be dispensed by a solenoid-operated liquid feeding device (Lehigh Valley Model 1577). These devices were calibrated so that each provided the same amount of liquid per activation. Three sets of constant-current ac shock sources (Lafayette Instruments, Model 5806) and complementary neon scramblers (Model 5820) were attached to the grid floors and metal walls of the chambers. Shock output was measured by an analogue voltmeter. Programming modules and a laboratory minicomputer, used for controlling stimuli and recording responses, were located in another part of the building.

\section{Procedure}

Barpress training and Phase 1 (P-1) adaptation to the CS. All rats were first trained to barpress on a variable-interval (VI 2-min) schedule for water reward until response rates were stable and asymptotic. All sessions were 50 min long and, with rare exceptions, occurred daily throughout the study. Each rat was then exposed to six presentations of the CS (the 7.5-W houselight) at the rate of two per day for 3 days. Four groups of 6 rats each were then formed on the basis of suppression to the sixth CS, using a randomized-blocks procedure. Responses were counted separately during the 3-min periods preceding (Period A) and during (Period B) the presentation of this CS (this procedure was followed with all $\mathrm{CS}$ exposures throughout the study). These data were formed into standard suppression ratios (SRs), B/(A +B), for each subject, following Annau and Kamin (1961); the SRs served as the values by which the groups were formed. One-way ANOVAs involving the four groups, applied separately to the SR data and to Period B and Period A response totals, produced $F$ values of unity or less.

For the following phases, all stimulus presentations were sched uled to occur randomly with respect to time, with the constraints that at least $5 \mathrm{~min}$ elapse between session onset and the initial stimulus exposure and that the minimum duration between US offset and onset be 10 min.

Phase 2: Fear acquisition. Three CS-US pairings (a pairing entailed exposure to a 3-min CS that overlapped and coterminated with a 1-sec, 1-mA US) were given to three of the four groups [designated Groups US(lo), US(hi), and PRF] on each of the next 3 days. All CS and US exposures were given during the VI 2-min, water-reward sessions and were programmed independently of barpresses. These pairings disrupted barpress rates somewhat. Five days of simple restabilization thus were given on the VI 2-min reward schedule. Then a single CS-US pairing was administered on each of the next 3 days for a total of 12 CS-US pairings over 11 days. A fourth group (Group RU) was given the same number of both stimuli, but in random order and in an explicitly unpaired relationship. These presentations were scheduled so that Group RU received US exposures at the same times as other groups. However, CS exposures were given independently of the US, with the stipulation that stimulus onsets and offsets were temporally separated by at least $5 \mathrm{~min}$.

Phase 3: Initial reductions in US density and intensity. Groups US(hi) and US(lo) continued to receive CS-US pairings on every trial; however, US intensity was lowered to $0.6 \mathrm{~mA}$ for the latter group. Group PRF (PRF = partial reinforcement) was scheduled to receive the US on only $50 \%$ of these trials. Since each trial for each subject of this group constituted the basis for random determination of US exposure, individual subjects received both different patterns and different numbers of shocks. Consequently, the numbers of USs given individual animals were $6,9,8,7,8$, and 
9, and the actual US density averaged $52 \%$. Group RU received the same number and intensity of CS and US presentations as did Group US(hi), but on a random and explicitly unpaired basis. Including 2 initial barpress restabilization days, this phase involved 15 trials (i.e., CS presentations) distributed over 11 days, that is, 2 restabilization days, two trials on each of Days 3 and 4, three on Day 5, one on Day 6, three on Day 7, barpress only on Days 8 and 9, and two trials on each of Days 10 and 11.

Phase 4: Intermediate reductions in US density and intensity. Fifteen more trials were given (over 11 days) to all groups. Groups US(hi) and US(lo) again were exposed to continuous CS-US pairings, but the US intensity was lowered for the latter to $0.4 \mathrm{~mA}$. The US density was programmed to decrease to about $20 \%$ (actual density was $17 \%$ ) for Group PRF, and resulted in 2, 2, 4, 1, 3, and 3 USs for the 6 subjects. Patterning of trials entailed two on Day 1, barpress stabilization on Days 2 and 3, two trials on each of Days 4 and 5 , one on Day 6, three on Day 7, two on Day 8 , none on Day 9, one on Day 10, and two on Day 11 .

Phase 5: Final reductions in US density and intensity. Fifteen trials were given over 10 days for this phase. Groups US(hi) and US(lo) again were administered continuous pairings, and the US intensity for the latter was further reduced to $0.3 \mathrm{~mA}$. The US density was programmed to be about $12.5 \%$ (actual density was $11 \%$ ), and resulted in 2, 1, 2, 2, 2, and 1 USs for the 6 rats. Patterning of trials entailed barpress stabilization on Days 1-2, one trial on Day 3, stabilization on Day 4, three on each of Days 5 and 6, one on Day 7, two on each of Days 8 and 9, and three on Day 10.

Extinction Phase E-1. All rats were exposed to three 3-min CSonly presentations on the baseline during each of six consecutive daily sessions. The choice of six sessions was determined by the criterion that extinction be carried out until at least one forwardconditioned group achieved a mean SR that was comparable to that of the control group. Because the experiment had been under way over 3 months by this time and joint maintenance of subject body weights and health was increasingly difficult to accurately regulate, the study was suspended temporarily and all rats were given access to food and water ad lib.

Extinction Phase E-2. Three months later, all rats were returned to their original $80 \%$ body-weight levels, restabilized on the VI 2min water-reward schedule, and given three CS-only presentations per session for an additional six consecutive daily sessions.

\section{RESULTS}

Response suppression to the CS and, separately, to the environment was evaluated in two ways. The first way involved standard SRs. Thus, suppression to the CS was indexed by the ratio $B /(A+B)$, as defined above. A second ratio was developed to evaluate suppression to environmental cues. Here, the number of responses emitted by each rat during Period $A$ of each trial served as the numerator. The denominator was this value summed with the number of barpresses that occurred during the Period $A\left(A^{\prime}\right)$ that preceded the last preconditioning $C S$ exposure of Phase 1 (a constant for each rat). This resulted in a ratio $A /\left(A^{\prime}+A\right)$ for each subject and trial of each phase.

A second way of indexing suppression to the CS was in terms of the number of responses that occurred during consecutive B periods. Suppression to the environment was evaluated in this second way by changes over trials

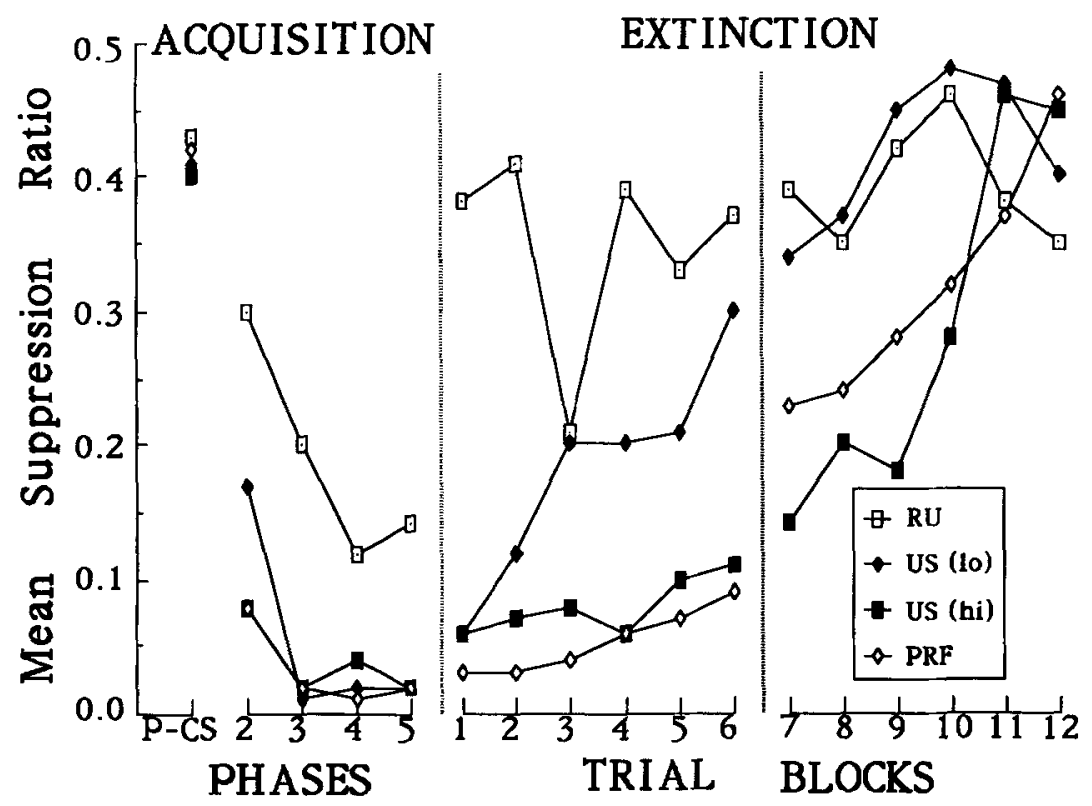

Figure 1. The left panel shows mean median suppression ratios, averaged by group and phases, for the last trial block of preconditioning CS exposures (P-CS); for all trial blocks of fear acquisition (2); and for the initial (3), intermediate (4), and final (5) phases involving stepwise reductions in US density (from $100 \%$ to $52 \%, 17 \%$, and $11 \%$ ) for Group PRF and in US intensity (from 1.0 to $0.6,0.4$, and $0.3 \mathrm{~mA}$ ) for Group US(lo). Group US(hi) received unchanging CS-US pairings and Group RU received unpaired, random CS and US exposures over Phases 2-5. The middle and right panels show group averages for each of six blocks of three trials for Extinction Phases E-1 and E-2 (see text). 
in response totals for consecutive A periods. Magnitude of suppression on these measures was referenced against the respective Period A and Period $B$ totals that were collected during the preconditioning phase $(P-1)$.

These two partially related measures of suppression are reported because of the important arguments of Hurwitz and Davis (1983) regarding possible problems of interpretation of the standard SR. They found that this index could give rise to spurious outcomes because of the changes in baseline responding (i.e., Period As) that often occur during conditioned suppression procedures. Use of response totals surmounts some of these problems and, as shown below, can result in conclusions somewhat different from those based upon SRs.

Medians for blocks of three trials were calculated for each subject for each set of derived and nonderived data; these medians constitute the basic unit for all of the analyses that follow. Mean median SRs depicting CS effects are shown in Figure 1 by group and by phase for Phases $1-5$, and by trial blocks for the two extinction phases. Figure 3 depicts the group environmental SRs for the same time frames. Figures 2 and 4 are displays of the mean median response totals for these acquisition and extinction phases for Periods B (Figure 2) and A (Figure 4). All statistical outcomes are judged against the .05 level of significance.

\section{Baseline}

Response totals (including those that occurred during the three CS exposures) for the second of the two preconditioning sessions of P-1 were subjected to a one-way
ANOVA involving all groups. The $F$ value approximated unity.

\section{CS Exposures}

\section{Suppression Ratios}

Preconditioning phase (Phase 1). Mean median SRs for the final block of three trials of this phase are shown by group in the far left panel of Figure 1. These means equal or exceed .40 and are tightly clustered together. When these means were subjected to an appropriate ANOVA, the result was an $F$ value of less than unity [as expected, the mean median ratios uniformly increased for each group over the two trial blocks of this phase; $F(1,20)$ $=11.1]$.

Acquisition and maintenance: Phases 2-5. The trialblock medians were averaged by phase for each subject and then by group, and these grand means are displayed in Figure 1 for the respective abscissa designations P-2, $P-3, P-4$, and P-5. The primary features of these data are that all but the random-unpaired subjects (Group RU) showed full suppression by the final CS-US acquisition trial block of P-2 (this is not revealed clearly in the figure because of the phasewise averaging procedure), and this suppression persisted across Phases 3-5. Group RU, although also suppressed relative to its $P-1$ baseline, appeared considerably less so for each of Phases 2-5 than did the other groups.

These data were analyzed with a 4 (groups) $\times 4$ (Phases 2-5) mixed-model ANOVA (the trial-blocks variable was omitted because it did not contribute significantly to any of the interaction variances). The $F$ values for groups
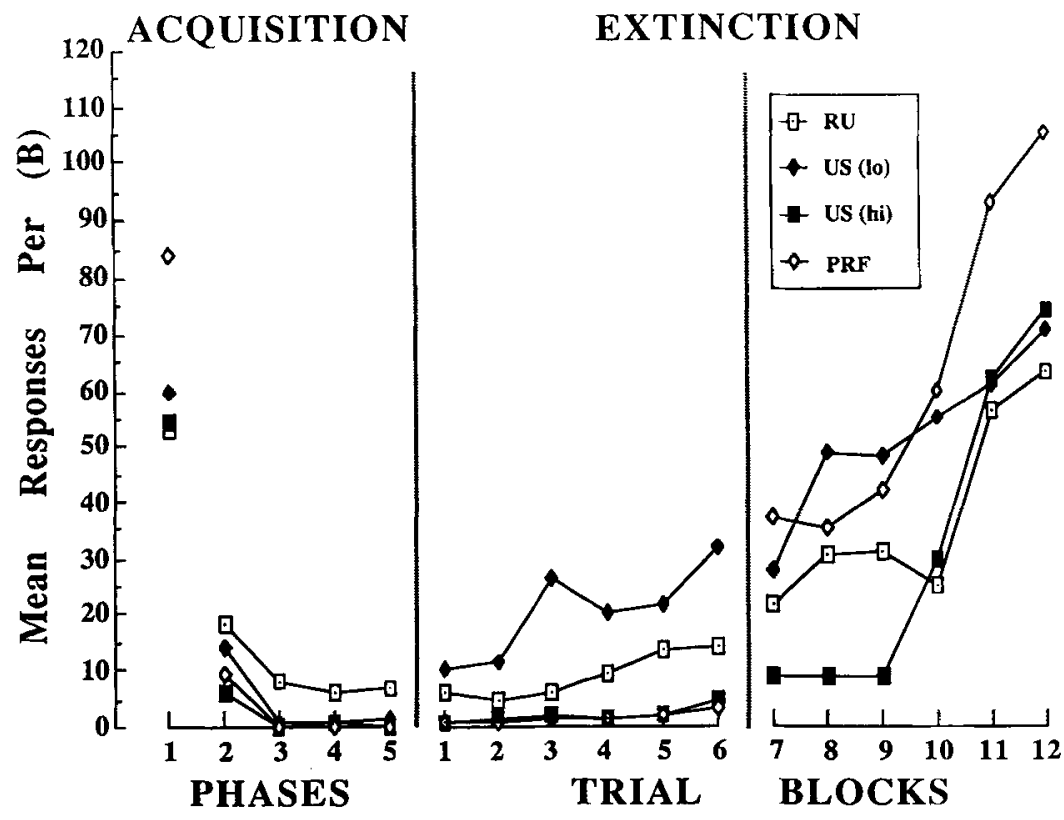

\section{EXTINCTION}

Figure 2. Responding during the CS (Period B), averaged in the same manner as for Figure 1. Fuase and group designations are the same as for Figure 1. 

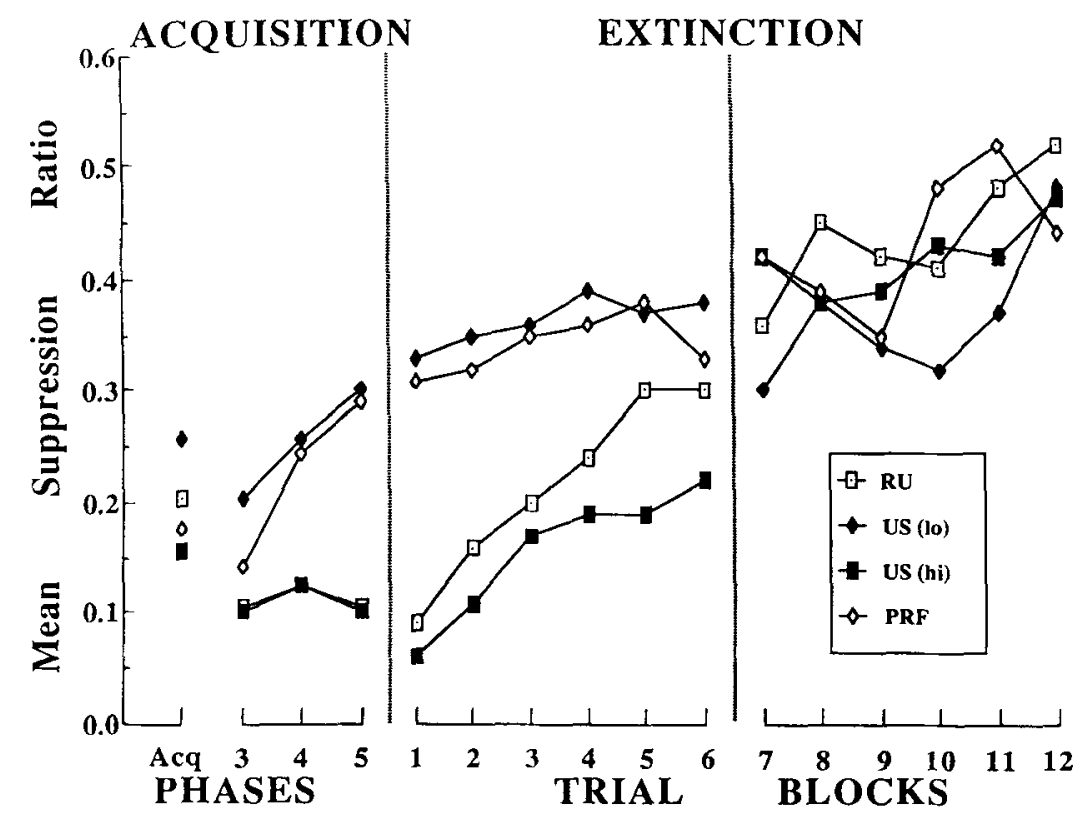

Figure 3. Mean median environmental suppression ratios, averaged by group and condition for acquisition (P-2) and Phases 3-5 (P-3, P-4, P-5), and by blocks of three trials for Extinction Phases E-1 and E-2. Group designations are the same as for Figure 1.

$[F(3,20)=4.6]$ and phases $[F(3,60)=23.1]$ were significant, but the $F$ for the interaction did not quite achieve normally accepted levels $[F(9,60)=2.0]$. All a priori follow-up comparisons between Group RU and each of the other three groups provided reliable $F$ values, whereas no $F$ s exceeded unity for pairwise comparisons among the three groups that were forward conditioned.
Extinction. The consequences of extinction differed for groups across both trial blocks and phases, thereby requiring the display of these data in greater detail than for Phases 1-5. Trial-block averages are shown for each group for the first (E-1) and second (E-2) extinction periods in the middle and right-hand panels of Figure 1. As shown, the SR average of Group RU for the initial

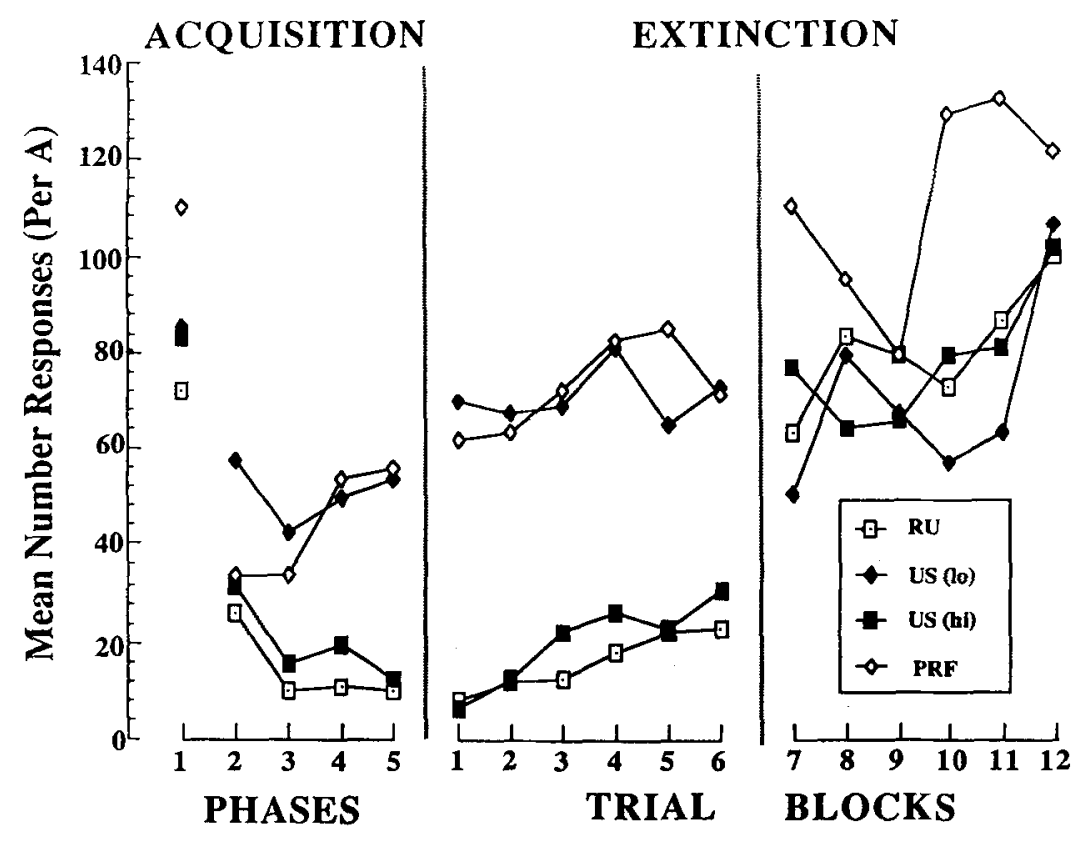

Figure 4. Responding during 3-min periods preceding CS exposures (Period A), averaged by group and condition in the same manner as for Figure 1. Group designations are the same as for Figure 1. 
trial block of E-1 was markedly larger than that for Phase 5, and remained relatively constant thereafter for this extinction set. By comparison, suppression stayed quite low and showed only a very modest decrease over these trial blocks for Groups US(hi) and PRF. However, Group US(lo) showed a gradual decrease in suppression over the trial sets of E-1, ranging from a performance indistinguishable from that of the latter two groups on Trial Block 1 to, by Trial Block 6, equivalence with Group RU. Recovery from suppression during E-1 thus occurred for only one of the three forward-conditioned groups.

Groups US(lo) and RU continued to show nonsuppressed and near-identical performances for all of the E-2 extinction phase. In contrast, Groups US(hi) and PRF evidenced relatively greater suppression on the early blocks of this phase, and only achieved full remission of fear by Trial Blocks 11 and 12. When subjected to a 4 (groups) $\times 2$ (Extinction Phases E-1 and E-2) $\times 6$ (trial blocks) mixed-model ANOVA, the $F$ values for all three main effects and three of the four interaction effects proved significant. The most important of these was the $F$ value for the three-way interaction $[F(15,100)=2.0]$.

Appropriate 4 (groups) $\times 6$ (trial blocks) follow-up analyses were conducted for each extinction phase. The $F$ ratio for groups was significant for Phase E-1 $[F(3,20)$ $=16.1]$ but not for blocks $[F(5,100)=2.04]$ or for the groups $\times$ blocks interaction $[F(15,100)=1.6]$. Nonetheless, the groups effect on each trial block (adjusted for inflated alpha error rates) yielded reliable $F$ s between controls and each of the forward-conditioned groups for the first two blocks and for all blocks involving Groups US(hi) and PRF. Furthermore, the differences between controls and Group US(lo) were not significant for Trial Blocks 3 and 6 of this phase, but the latter group was significantly less suppressed than the two other forward-conditioned groups by Trial Block 6 .

The pattern of results for the second extinction phase, E-2, was different from that for E-1 in that the main effect for groups was not significant and the $F$ values for blocks and the groups $\times$ blocks interaction $[F(15,100)$ $=3.5]$ were. Pairwise comparisons for each block revealed that Groups US(hi) and PRF, although not different from one another, were significantly more suppressed than were the controls and the US(lo) rats (which also were not different from one another) for the first four, but not the last two, blocks of this phase.

\section{Response Totals: Period B}

Preconditioning, acquisition, and maintenance phases (P-1 through P-5). These data are shown as averages for each group and phase in Figure 2. Preconditioning (P-1) response rate during the $C S$, although arithmetically higher for Group PRF than for the others, did not differ statistically across groups $[F(3,20)=2.0]$. The same basic pattern of suppression as revealed by the SR data of Figure 1 can be seen for Period B response rates for Phases 2-5 in Figure 2, in that Group RU performed at an over- all higher rate than did the other groups. However, the overall difference in Period B suppression between controls and the forward-conditioned groups was observably smaller than that depicted by the SR data of Figure 1. This is because Group RU showed a general suppression of responding, whether in the presence or absence of the CS. Thus, when made relative as a ratio, suppression for this group in the presence of the CS appeared to be much less than it actually was. In contrast, Period B responding was essentially zero for the other groups, and wide variations in suppression to the environment thereby exerted little influence on the magnitude of the SR. These observations were supported by a nonreliable $F$ value for the groups effect $[F(3,20)=1.4]$ in a 4 (groups) $\times 4$ (Phases 2-5) mixed-model ANOVA. However, a priori pairwise comparisons involving Group RU (adjusted for inflated alpha rates) resulted in reliable differences in all cases [lowest $t(20)=2.51$ for the comparison with Group US(lo)].

Extinction Phases E-1 and E-2. Because the groups $X$ phases $X$ trial blocks analysis resulted in several reliable interactions with phases, the outcomes for each phase are considered separately. The data for Phase E-1 differed from those involving SRs in that Group US(lo) evidenced even greater recovery of responding over trial blocks than did Group RU. These two groups, as seen in Figure 2, responded at higher levels than did either Group PRF or Group US(hi). The latter groups remained almost completely suppressed throughout this phase. Pairwise comparisons involving all possible combinations, adjusted for inflated alpha rates, revealed that Group US(lo) responded reliably more, overall, during the CS than did Group RU $[t(20)=3.7]$, and that Group RU responded more than did either of the remaining groups for each trial set [all $t \mathrm{~s}(20)>2.3]$.

All groups showed recovery from suppression during the second extinction phase, but at different rates. This was reflected in a reliable groups $\times$ blocks interaction $[F(15,100)=2.0]$. Group US(hi) clearly evidenced less responding than did the other three groups for the initial three trial blocks. Group US(hi) was similar to Group RU for Trial Block 4 (both of which were more suppressed than the other two for this trial set), and all groups responded at preconditioning levels by Trial Blocks 5 and 6 (Group PRF arithmetically showed more responding on these trial blocks, but not significantly more). This pattern of responding was supported by appropriate trial $x$ trial statistical comparisons.

\section{Environmental Suppression}

\section{Suppression Ratios}

Acquisition and maintenance: Phases 2-5. As shown in Figure 3, phasewise SR averages were relatively low and were about the same for all groups for Phase 2 (acquisition), but gradually recovered over Phases 3-5 for Groups PRF and US(lo). By comparison, Groups RU and US(hi) remained unchanged and quite suppressed throughout these periods. A separate 4 (groups) $\times 4$ (trial blocks) ANOVA was performed on the acquisition (Phase 2) data 
and, with the exception of trial blocks, no $F$ values achieved significance for this analysis $(F \mathrm{~s}<1.0)$. However, a 4 (groups) $\times 3$ (Phases 3-5) $\times 5$ (trial blocks) ANOVA yielded a significant groups $x$ phases interaction, thereby necessitating separate phase $\times$ phase follow-up analyses. Thus, 4 (groups) $\times 5$ (blocks) ANOVAs were performed for each of Phases 3-5. Only blocks effects emerged as significant for Phases 3 and 4, but both the blocks $[F(4,80)=4.6]$ and groups $[F(3,20)$ $=4.2]$ effects proved statistically significant for Phase 5 . A posteriori pairwise follow-up comparisons for the Phase 5 data showed that Groups PRF and US(lo) were less suppressed than were Groups RU and US(hi), but that the members of each pair were not statistically distinguishable from one another.

Extinction. The middle panel of Figure 3 shows that the differences in suppression between pairs of groups for the last maintenance phase (P-5) remained the same throughout the early trial blocks of the first extinction phase (E-1). This difference essentially disappeared by the final trial sets of Phase E-1 because of the gradual attenuation (over blocks) in suppression by Groups RU and US(hi) relative to the basically unchanged and only nominal suppression of the other two groups. This clustering of mean median group suppression ratios carried over to the second extinction phase (E-2, last panel of Figure 3), even though all groups evinced even less overall suppression than during the first extinction phase.

These extinction data were subjected to a 4 (groups) $\times 2$ (extinction phases) $\times 6$ (blocks) ANOVA, resulting in a significant groups $\times$ extinction phase interaction effect $[F(3,20)=4.6]$. Separate phasewise analyses produced significant $F$ values of 3.3 and 8.8 for groups and blocks for Phase E-1 and for blocks for Phase E-2, but neither interaction proved reliable. Follow-up pairwise comparisons for Phase E-1, adjusted for inflated alpha rates, produced significant $F$ s for contrasts between either of Groups PRF and US(lo) and either of Groups RU and US(hi), but not for contrasts within either of these pairs.

Since the arithmetic pattern and statistical outcomes for the Period A response totals approximated those for the environment SRs, they do not require further discussion.

\section{DISCUSSION}

These data showed that fear could be acquired and maintained within a classical conditioning paradigm in the presence of certain features of the CS and US that normally are part of avoidance training. Fear conditioning per se was demonstrated through the (somewhat) less suppressed performance of Group RU than of Group US(hi) for Phases 2-5. Since both groups were given the same stimulation, these phasewise variances in response rate may be attributed to the different contingency relationships between the CS and US for each. All forwardconditioned groups displayed complete suppression to the CS on both relative (SR data) and absolute (Period B to- tals) bases by the end of acquisition (Phase 2). This full suppression was sustained thereafter even though the final 15 trials of Phase 5 entailed an average of only 1.7 US exposures for one of these groups (Group PRF) and a near-threshold US that did not produce observable abient reactions for another [Group US(lo)].

The outcome associated with Group PRF was different from that obtained by Gibbs, Latham, and Gormezano (1978), who found attenuated performance of the nictitating membrane conditioned response following a somewhat similar conditioning (continuous reinforcement), maintenance (partial reinforcement), and extinction procedure. One factor that may partially account for this variance in outcomes is that, along with quite different response preparations and systems, the present study involved stepwise reductions in US density, whereas that of Gibbs et al. (1978) did not. Instead, the present findings for Group PRF more closely resembled those of Starr and Mineka (1977), Linden (1969), and others that have evaluated the effects of partial reinforcement on conditioned suppression. Thus, even though Group PRF received many fewer (24) CS-US pairings than the 57 given Group US(hi), both groups performed identically to the CS throughout the study. This marked suppression by Group PRF, persisting as it did throughout extended classical extinction testing, was most like Starr and Mineka's (1977) Group YAL, one of the fear-conditioned groups that showed full suppression after 27 consecutive exposures to a much shorter duration CS.

Parenthetically, Starr and Mineka (1977) employed a control in their study for the number of USs (the groups designated as YFC in their report) given to their partialreinforcement groups, whereas the present study employed a control [Group US(hi)] for number of trials given to its Group PRF subjects. In neither study did the use of these control conditions result in any differences between the controls and their respective experimental groups in terms of suppression during testing, thereby providing nominal convergent support for the robustness of the present finding. That is, the fully suppressive (to the CS) effect of partial reinforcement during fear training now has been shown in connection with two different control conditions. (The present study differed from that of Starr and Mineka in other ways as well. The present study included many more days of conditioning and extinction, and their study involved variable CS durations.)

Another finding of the present study was that 57 CS-US pairings with a decreasingly intense US [Group US(lo)] resulted in relatively rapid extinction of suppression. This outcome shows that the effects of partial reinforcement on the extinction of a classical fear reaction are not due to alterations per se of a major feature of the US during training. Clearly, the nature of the alteration is important as well. This finding of rapid extinction for Group US(lo) would be especially interesting if it could be shown somehow that the graded reduction in shock intensity was functionally equivalent to the reduced number of USs given to Group PRF. As it turns out, indirect informa- 
tion in this connection is provided by the present study. If conditioning to situational cues can be taken as an independent (of the CS) indication of US potency, then the similar suppression functions of Groups PRF and US(lo) to environmental stimuli suggest the possibility of functional equivalence for these two US regimens. This notion, of course, leaves unanswered the question of why fear loading to the CS, as revealed during the extinction phases, nonetheless was so different for these two groups (discussed below). A related finding was that when suppression to both the CS and the environment was taken into account, the two groups that were exposed to the smallest number or lowest intensity of shock(s) throughout acquisition and maintenance displayed the greatest differential responding to the $C S$ in that they showed the larger differences between respective rates of responding to the environment and to the CS.

A final finding of this study pertained to the somewhat different outcomes generated by the derived and nonderived measures. For example, Period B suppression for Group RU was both more marked and more arithmetically like that of the Period B suppression of the feartrained rats for Phases 2-5 than was apparent when SRs were used for purposes of comparison. Furthermore, suppression was actually greater on the Period B (but not the SR) measure for Group RU than for one of the feartrained groups [Group US(lo)] for both of the extinction phases. These observations reaffirm the conclusions of Hurwitz and Davis (1983) that the outcomes of conditioned suppression studies can differ both in degree and in kind depending upon whether or not an adjustment is made for differences in baseline responding through application of Annau and Kamin's (1961) SR formula.

There has been a long-standing interest in the interplay between the amount of fear that is associated with a discretely presented CS and that which may become connected to the ambient features of the situation in which conditioning takes place (Balsam \& Tomie, 1985; McAllister \& McAllister, 1962; Callen, McAllister, \& McAllister, 1984). Certain of the present data may bear on at least one of these interests, namely, whether the amount of fear that becomes associated with situational cues is different according to whether it is signaled or unsignaled (McAllister, McAllister, Weldin, \& Cohen, 1974; OdlingSmee, 1975). For example, Group RU and Group US(hi) displayed identical amounts of suppression to the situational cues for all phases of the study, even though shock was signaled for only one of them. When contrasted with the less suppressed responding of the other two groups (who were given signaled USs) to the ambient environmental cues, one is left with the conclusion that the primary determinant of attenuated performance to the situational cues in the present study apparently is traceable to variations in aspects of the US rather than whether or not a CS was involved. Noteworthy also is that, although variations in A-period response-rate averages were quite large between the three forward-conditioned groups, each nonetheless differed from controls in showing nondistinguishable, complete suppression to the CS throughout
Phases 2-5. This finding seems to show that suppression to the CS may be independent of suppression to the environment as well (although qualification may be necessary here on the grounds of insensitive measurement procedures).

Several theories have at least some bearing on the present findings. For example, a fear-summation hypothesis (Hendry, 1982; Reberg, 1972), according to which fear of the context may sum with fear of the CS, may account for the differences observed during extinction between certain of the groups. Here, the variations in resistance to extinction of fear of the CS might be attributable partly to differences in the amount of fear of the CS, of situational cues, or both that was available for summation. (Obviously, this theory does not account for the differences in associative loading of fear that may have occurred separately in response to the CS and to these situational cues.) That all of the fear-conditioned groups were equivalently suppressed during the preextinction Phases 2-5 may be explained by the possibility that the fear measurement indices were insensitive. Both the SR and Period B measures would be incapable of resolving differences in fear if the least fearful group nonetheless exceeded the amount that resulted in full response suppression. In this case, differences in fearfulness could be detected only during extinction, when the US was no longer given. Regrettably, the combined notions of fear summation and insensitive measurement probably cannot account for the equivalent suppression by Groups PRF and US(hi) to the CS during extinction, since the former group was much more fearful than the latter group of the situation cues during these periods.

The Rescorla-Wagner (1972) contingency theory of classical conditioning also is consistent with the present finding of relatively more rapid extinction for Group US(lo). However, this theory does not fare well in explaining the effects reported for Group PRF. According to the formula that Rescorla and Wagner $(1972$, p. 85) developed in this connection, associative strength to the partially reinforced CS appears to be decremented as a function of the proportion of reinforced trials for each phase. If the different US density percentages-namely, $.52, .17$, and .11 for Phases 3-5-are inserted into their formula, associative strength to the CS should decrease from unity (Phase 2) to about .70 for Phase 3, to around .29 for Phase 4, and to .20 for Phase 5. Indeed, this theory appears to generate the prediction that CS associative strength at the outset of extinction should be about the same for Groups PRF and US(lo). Assuming no differential carry-over from the conditioning to the extinction phases of unique nonassociative influences on performance, the prediction by the PRF aspect of this model of conditioning appears to be inconsistent with the outcome for Group PRF. Other, somewhat similar theories (see the neo-S-R theory of Spence, 1956) encounter the same difficulty in explaining the present partialreinforcement effect.

Thus, although several current theories of conditioning may account for most or all of the present findings 
for Groups RU, US(hi), and US(lo), many seem unable to account for the fact that Groups PRF and US(hi) showed equivalent fear of the CS but not of the situational cues. One possible explanation for this equivalent fear of the CS may have to do with the ingredient of US unpredictability that occurred for Group PRF in connection with the environment plus the CS but not with the environment alone. On the presumption that such unpredictability may be highly unpleasant (see Badia, Coker, \& Harsh, 1973; Paré \& Livingston, (1973) or surprising (Kamin, 1969), the result might be augmentation of US effectiveness in connection with the CS (but not the environment). Thus, the present partial-reinforcement data might best be reconciled by a combination of either neo-S-R or RescorlaWagner theory and unpredictability theory.

\section{REFERENCES}

Annau, Z., \& Kamin, L. J. (1961). The conditioned emotional response as a function of intensity of the US. Journal of Comparative \& Physiological Psychology, 54, 428-432.

Badia, P., Coker, C., \& HARsh, J. (1973). Choice of higher density signalled shock over lower density unsignalled shock. Journal of the Experimental Analysis of Behavior, 20, 47-55.

Balsam, P. D., \& Tomie, A. (1985). Context and learning. Hillsdale, NJ: Erlbaum.

Brown, J. S. (1961). The motivation of behavior. New York: McGraw-Hill.

Callen, E. J., McAllister, W. R., \& McAllister, D. E. (1984). Investigations of the reinstatement of extinguished fear. Learning \& Motivation, 15, 302-320.

Gibbs, C. M., Latham, S. B., \& Gormezano, I. (1978). Classical conditioning of the rabbit nictitating membrane response: Effect of reinforcement schedule on response maintenance and resistance to extinction. Animal Learning \& Behavior, 6, 209-215.

HENDRY, J. S. (1982). Summation of undetected excitation following extinction of the CER. Animal Learning \& Behavior, 10, 476-482.

HuRwitz, H. M. B., \& Davis, H. (1983). The description and analysis of conditioned suppression: A critique of the conventional suppression ratio. Animal Learning \& Behavior, 11, 383-390.

KAMIN, L. J. (1969). Predictability, surprise, attention and conditioning. In B. A. Campbell \& R. M. Church (Eds.), Punishment and aversive behavior (pp. 279-296). New York: Appleton-Century-Crofts.

Kamin, L. J., Brimer, C. J., \& BLACK, A. H. (1963). Conditioned suppression as a monitor of fear to the $\mathrm{CS}$ in the course of avoidance training. Journal of Comparative \& Physiological Psychology, 56, 497-501.

Kimble, G. A. (1961). Hilgard and Marquis' conditioning and learning (2nd ed.). New York: Appleton-Century-Crofts.

LINDEN, D. R. (1969). Attenuation and reestablishment of the CER by discriminated avoidance conditioning in rats. Joumal of Comparative \& Physiological Psychology, 69, 573-578.

MCAllister, W. R., \& MCAllister, D. E. (1962). Role of the CS and of apparatus cues in measurement of acquired fear. Psychological Reports, 11, 749-756.

McAllister, W. R., McAllister, D. E., Weldin, G. H., \& Cohen, J. M. (1974). Intertrial interval effects in classically conditioning fear to a discrete conditioned stimulus and to situational cues. Journal of Comparative \& Physiological Psychology, 87, 582-590.

MinekA, S. (1985). The frightful complexity of the origins of fears. In F. R. Brush \& J. B. Overmier (Eds.), Affect, conditioning, and cognition: Essays on the determinants of behavior (pp. 55-74). Hillsdale, NJ: Erlbaum.

MineKa, S., CoOK, M., \& Miller, S. (1984). Fear conditioned with escapable and inescapable shock: The effects of a feedback stimulus. Journal of Experimental Psychology: Animal Behavior Processes, 10, 307-323.

MowRER, O. H. (1947). On the dual nature of learning-A reinterpretation of 'conditioning' and 'problem solving.' Harvard Educational Review, 17, 102-148.

MOWRER, O. H. (1960). Learning theory and behavior. New York: Wiley.

ODLING-SMEe, F. J. (1975). The role of background stimuli during Pavlovian conditioning. Quarterly Journal of Experimental Psychology, 27, 201-209.

PARE, W., \& Livingston, A., JR. (1973). Shock predictability and gastric secretion in the chronic gastric fistula rat. Physiology \& Behavior, 11, $521-526$.

REBERG, D. (1972). Compound tests for excitation in early acquisition and after prolonged extinction of conditioned suppression. Learning \& Motivation, 3, 246-258.

Rescorla, R. A., \& Solomon, R. L. (1967). Two-process learning theory: Relationships between Pavlovian conditioning and instrumental learning. Psychological Review, 74, 151-182.

Rescorla, R. A., \& Wagner, A. R. (1972). A theory of Pavlovian conditioning: Variations in effectiveness of reinforcement and nonreinforcement. In A. H. Black \& W. F. Prokasy (Eds.), Classical conditioning II: Current research and theory. New York: AppletonCentury-Crofts.

STARR, M. D., \& Mineka, S. (1977). Determinants of fear over the course of avoidance learning. Learning \& Motivation, 8, 332-350.

(Manuscript received September 5, 1986; revision accepted for publication May 1, 1987.) 